\title{
Politique
}

\section{Répertoire des mémoires de maîtrise, 1983}

Numéro 5, hiver 1984

Femmes et pouvoir

URI : https://id.erudit.org/iderudit/040458ar

DOI : https://doi.org/10.7202/040458ar

Aller au sommaire du numéro

Éditeur(s)

Société québécoise de science politique

ISSN

0711-608X (imprimé)

1918-6584 (numérique)

Découvrir la revue

Citer ce document

(1984). Répertoire des mémoires de maîtrise, 1983. Politique, (5), 166-170.

https://doi.org/10.7202/040458ar

Ce document est protégé par la loi sur le droit d'auteur. L'utilisation des services d'Érudit (y compris la reproduction) est assujettie à sa politique d'utilisation que vous pouvez consulter en ligne.

https://apropos.erudit.org/fr/usagers/politique-dutilisation/
Cet article est diffusé et préservé par Érudit.

Érudit est un consortium interuniversitaire sans but lucratif composé de l’Université de Montréal, l'Université Laval et l'Université du Québec à Montréal. Il a pour mission la promotion et la valorisation de la recherche. https://www.erudit.org/fr/ 


\section{NOM DE L'AUTEUR(E)}

Bérard, Jacques

Berthiaume, Jean-Luc

Boivin, Marc

Desjardins, Jacques

Dubuc, Alain

Haouchine, Ali

Joseph, Jean-Serge

Kane, Michel

Lairini, Najib

Lefebvre, Michel

Maillé, Chantal

Mallette, Yves

Marois, Michel

Ouadraogo, Noraogo

Vincent

\section{TITRE}

UNIVERSITÉ

"Continentalisme et fragmentation économique au Canada: essai de théorisation et éléments de démontration"

«La réforme municipale du gouvernement du Parti québécois: une analyse de la décentralisation"

"La réforme municipale du gouvernement du Parti québécois une analyse de décentralisation"

"La politique de concession des droits privés de location en matière de chasse et de pêche au Québec, de 1960 à 1977"

«La réforme électorale au Québec (1976-1981)»

"L'état et les travailleurs: le cas de l'Algérie, de 1965 à 1979"

"Le développement dépendant: le cas du Mexique, de 1964 à 1980"

«La stratégie de prise du pouvoir du parti communiste français, 19621979"

«Étude critique des textes régissant le «Discours sur le nouvel ordre économique international" "

"Analyse des politiques québécoises de sélection et d'intégration en matière de réfugiés"

"Étude anthologique des écrits théoriques du féminisme»

"L'affaire Lin Biao: une explication à partir de l'approche selon les groupes d'intérêt"

«L'influence des fonctionnaires dans la réforme du système québécois de santé-sécurité au travail»

"L'impact du régionalisme africain sur le groupe des travailleurs à l'Organisation Internationale du Travail (O.I.T.)"
Université de Montréal

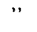

,

$"$

"

"

,

,

,

,

",

,

Université de Montréal

",

"

,

,

,

, 
Sarrasin, Hélène

Schanck, Daniel

Sheils, Gwen

Taliadoros, Georges

Beauchemin, Pierre

Bissonnette, Rita

Guay, Jean

Mestiri, Brahim

Theauvette, Carole

Audinot, Marie-Pascal

Bérubé, Pierre/Bischoff, Manfred

Bouchard, Pier/Vézina, Sylvain

Brasier, Fabrice

Cadotte, André

Charland, Roger

Collin, Jean-Pierre
«La pratique des intellectuels dans les groupes populaires: le cas du Front d'action populaire en réaménagement urbain"

"L'entreprise d'État comme instrument de politique industrielle du Québec. Les cas Rexfor et Soquem"

«La démocratie de participation: la voie à suivre pour les relations de travail du Québec?»

"La culture politique arabo-islamique et la naissance du nationalisme algérien (1830-1962)"

"L'activité des organisations internationales: le cas du Programme des Nations-Unies pour le Développement”

«La régionalisation municipale au Québec 1960-1980: visées technocratiques et résistances locales"

«Les différentes composantes du comportement politique analysées par W. Reich"

«La pensée politique et sociale de Habbib Bourguiba»

«La domiciliation de la Constitution canadienne: l'affrontement de deux conceptions du fédéralisme"

"La politique agricole et la question paysanne au Sénégal (1960-1980)"

«Le rôle du politique dans la production industrielle, 1860-1920. Une contribution critique à l'analyse de Marx"

«Le développement bureaucratique du secteur des affaires sociales au Québec»

«La communauté économique européenne et le Tiers-Monde: néo-colonialisme ou amorce d'un nouvel ordre économique international? Le cas de la première convention de Lomé (1975-1980)"

«Préalables pour une analyse du procès de formation de l'État au Canada»

"Karl Korsch. Révolution, contre-révolution et crise du marxisme»

«Pouvoir municipal et enjeux politiques locaux dans la paroisse de Montréal, de 1871-1921. (Étude de la formation des banlieues, de l'incorporation de nouvelles municipalités et de leur activité réglementaire)»
Université d'Ottawa

Université du

Québec

à Montréal

", 
Desbiens, Pierre

Doua, Blé Siméon

Ducheine, Viviane

Dufresne, Danièle

Durocher, Pierre

Fournier, François/

Villeneuve, Daniel

Lanthier, Richard

Racine, Daniel

Sadria, Modj-Ta-Ba

Sénécal, Francine

Thivierge, Robert

Beaulieu, Suzanne

Milot, Pierre

Piard, Frantz

Agondjo, Jean-Richard

Alami-Binani, Abdelilah

Beaudet, André
«Introduction au couple État-Économie»

"Décentralisation et contrôle en organisation (gouvernementale)"

"L'information et le Tiers-Monde: une analyse du déséquilibre de la circulation des nouvelles entre le Nord et le Sud"

«Les enseignantes au Québec des débuts à 1973 et dans le syndialisme enseignant (C.E.Q.)"

«L'évaluation de la politique sociale du logement du gouvernement fédéral canadien, 1964-1979",

«La dynamique des transformations constitutionnelles au sein de l'État canadien: le déploiement du centralisme fédéral contre le Québec et les pouvoirs provinciaux (1980-1981)"

«Espace, capital et transport (vers une théorie marxiste du rôle et de l'importance de la sphère des transports dans le développement du mode de production capitaliste»

«La loi de la qualité de l'environnement: une loi défensive et marginale (analyse socio-politique)"

«L'Arabie de 1900 à 1932: vers la formation d'un État Saoudien"

"Les politiques gouvernementales en éducation des adultes et les initiatives populaires"

«Le rôle de la municipalité de Salaberry de Valleyfield dans le développement industriel local de 1960 à 1970"

"Évaluation du rôle des pays exportateurs de pétrole»

"Généalogie du discours marxiste-léniniste au Québec (1972-1982)"

«Le non-alignement dans la politique internationale: essai sur la pratique algérienne du non-alignement"

"Le conflit de la corne orientale de l'Afrique»

"L'enseignement au Maroc - Le financement mixte du secondaire»

«La fonction tribunitienne et les partis nationalistes-souverainistes: les cas du Rassemblement pour l'Indépendance Nationale et du Scottish National Party»
Université

Québec

à Montréal

du

,

,

Université Laval

,

, 
Binet, Lise

Bouchard, Yvan

Bouffard, Denys-Paul

Duhaime, Gérard

Gauthier, Johanne

Godin, Rita

Gravel, Rénald

Guay, Luc

Guihede, Ange

Karzazi, Hassan

Lavoie, Marie

Ledoux, Geneviève

Massé, Hélène

Mulemeri-Kanamby, Paul
"La naturalisation des rapports sociaux: l'exemple de l'institution de maternité"

"L'avancement du personnel professionnel de la fonction publique du Québec: le cas de l'avancement à la classe I des attachés d'administration"

«Nationalisme et choix de politiques gouvernementales. Le cas du développement et de l'utilisation des ressources hydro-électriques au Québec entre 1979 et 1981"

«Espace politique et logements sociaux au Nouveau-Québec Inuit»

«État providence, centralisation et décentralisation politico-administratives: le cas des équipements collectifs dans le domaine des services sociosanitaires au Québec»

«Le développement d'un tiers parti: Le Parti acadien de son origine à 1982"

"La théorie économique du nationalisme: une application aux politiques de l'enseignement supérieur au Québec"

"Étude comparative de modèles générationnels. La question nationale au Québec 1960-1980"

"La diversification commerciale et le phénomène de la dépendance: la Côte d'Ivoire de 1965 à 1980"

"Conflits sahariens et évolution politique au Maroc de 1956-1981"

"La réforme électorale: évaluation critique et scénarios de réforme"

"L'accès à l'information gouvernementale: analyse politique de la proposition de loi de la commission d'étude Paré au Québec»

"De l'autonomie d'action syndicale des femmes à la récupération patriarcale une étude de cas: La fédération catholique des institutrices rurales de 1936 à 1953"

"La politique internationale du Congo-Kingshasa de 1960) à 1965: essai d'explication de l'élchec de cette politique fondée sur le nonalignement et le panafricanisme"
Université Laval

,

"

",

",

",

Université Laval

"

, 
N'Diaye, Cheikh Amadou Tidiane

Poirier, Guy-J.

Riverin, Jean-Paul

Savas, Daniel

Al-Yassini, Ayman

Soare, Richard

Walker, Stephen
«Le protocole no 3 dans la convention de Lomé (1975-1980): essai d'analyse critique"

«Les politiques d'exportation de gaz naturel de l'Office national de l'énergie de 1959 à 1979"

"Analyse conjoncturelle et structurelle de la crise universitaire de mai $1968 "$

«Le discours politique et le nationalisme québécois»

"The relationship between religion and state in the Kingdom of Saudi Arabia»

Université

McGill

"The epistomological roots of John Locke's theory of toleration"

"A pragmatic approach to social enquiry" 